\title{
Peritoneal and pericardial involvements of multifocal african histoplasmosis on sever undernutrition
}

\author{
Nomtondo Amina Ouédraogo ${ }^{1,2}$, Anicette Alida Kouassi ${ }^{1}$, Muriel Sidnoma Ouédraogo ${ }^{1,2}$, \\ Kounpielime Sosthène Somda ${ }^{2,3}$; Gilbert Patrice Tapsoba ${ }^{1,2}$, \\ Angèle Ouangre/Ouédraogo ${ }^{1}$, Ismaël Diallo ${ }^{2,4}$, Adama Zidaa, ${ }^{2,5}$ Fagnima Traore ${ }^{6}$, \\ Djounitana Djimtibaye ${ }^{1}$, Danielle Belemsigri', Valentin Nobila Yaméogo ${ }^{2,7}$, \\ Nessine Nina Korsaga/Somé2,8, Fatou Barro/Traoréé,9, Pascal Niamba ${ }^{1,2}$, Adama Traoré1,2
}

${ }^{1}$ Department of Dermatology-Venereology of Yalgado Ouédraogo University Hospital (YO UH), Ouagadougou, Burkina Faso, ${ }^{2}$ Health Science Training and Research Unit, Joseph Ki-Zerbo University, Ouagadougou, Burkina Faso, ${ }^{3}$ Department of Gastro-Hepato-Enterology of YO UH, Ouagadougou, Burkina Faso, ${ }^{4}$ Department of Infectious and Tropical Diseases of YO UH, Ouagadougou, Burkina Faso, ${ }^{5}$ Department of Parasitology and Mycology of YO UH, Ouagadougou, Burkina Faso, ${ }^{6}$ Department of Dermatology, Regional University Hospital of Ouahigouya, Burkina Faso, ${ }^{7}$ Department of Cardiology of YO UH, Ouagadougou, Burkina Faso, ${ }^{8}$ Dermatology Unit, Boulmiougou District Hospital, Ouagadougou, Burkina Faso, ${ }^{9}$ Department of Dermatology of Tingandogo University Hospital, Ouagadougou, Burkina Faso

Corresponding author: Nomtondo Amina Ouédraogo, MD, E-mail: nomtondo2000@yahoo.fr

\begin{abstract}
African histoplasmosis is rare in our country. We report a case of multifocal African histoplasmosis with peritoneal and pericardial involvement. A 27 year old man, a gold digger, consulted for disseminated papular and nodular lesions that had been evolving for 3 months. On examination, cachexia (body mass index 15.19kg/m2), cold abscesses all over the integument, umbilicated papules predominating on the face and ascites were noted. Paraclinical investigations showed neutrophilia, anaemia, hypoprotidemia, exudative ascites, pericarditis, severe pulmonary arterial hypertension, large 8-shaped yeasts of Histoplasma capsulatum var. duboisii on direct examination of the mycological specimen, confirming African histoplasmosis. Good clinical outcome was achieved with itraconazole. Severe malnutrition was probably a risk factor for dissemination of the disease
\end{abstract}

Key words: Visceral involvement, undernutrition, itraconazole, Burkina Faso

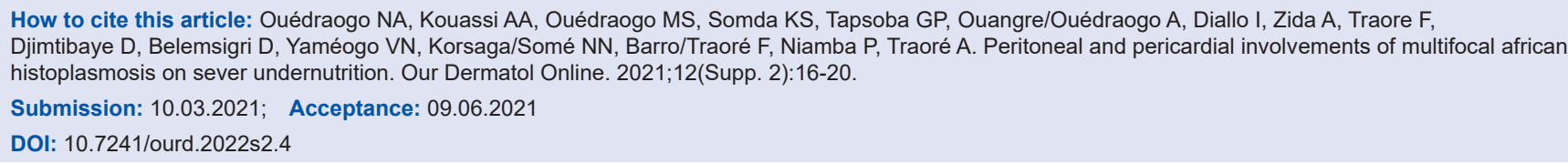




\title{
Atteintes péritonéale et péricardique d'une histoplasmose africaine multifocale sur une dénutrition sévère
}

\author{
Nomtondo Amina Ouédraogo ${ }^{1,2}$, Anicette Alida Kouassi ${ }^{1}$, Muriel Sidnoma Ouédraogo ${ }^{1,2}$, \\ Kounpielime Sosthène Somda ${ }^{2,3}$; Gilbert Patrice Tapsoba ${ }^{1,2}$, \\ Angèle Ouangre/Ouédraogo ${ }^{1}$, Ismaël Dialloº,4, Adama Zida ${ }^{2,5}$, Fagnima Traore ${ }^{6}$, \\ Djounitana Djimtibaye ${ }^{1}$, Danielle Belemsigri', Valentin Nobila Yaméogo ${ }^{2,7}$, \\ Nessine Nina Korsaga/Somée, ${ }^{2,8}$ Fatou Barro/Traoré2,9, Pascal Niamba ${ }^{1,2}$, Adama Traoré1, 2.
}

${ }^{1}$ Department of Dermatology-Venereology of Yalgado Ouédraogo University Hospital (YO UH), Ouagadougou, Burkina Faso, ${ }^{2}$ Health Science Training and Research Unit, Joseph Ki-Zerbo University, Ouagadougou, Burkina Faso, ${ }^{3}$ Department of Gastro-Hepato-Enterology of YO UH, Ouagadougou, Burkina Faso, ${ }^{4}$ Department of Infectious and Tropical Diseases of YO UH, Ouagadougou, Burkina Faso, ${ }^{5}$ Department of Parasitology and Mycology of YO UH, Ouagadougou, Burkina Faso, ${ }^{6}$ Department of Dermatology, Regional University Hospital of Ouahigouya, Burkina Faso, ${ }^{7}$ Department of Cardiology of YO UH, Ouagadougou, Burkina Faso, ${ }^{8}$ Dermatology Unit, Boulmiougou District Hospital, Ouagadongou, Burkina Faso, ${ }^{9}$ Department of Dermatology of Tingandogo University Hospital, Ouagadougou, Burkina Faso

Corresponding author: Nomtondo Amina Ouédraogo, MD, E-mail: nomtondo2000@yahoo.fr

\section{RÉSUMÉ}

L'histoplasmose africaine est rare dans notre pays. Nous rapportons un cas d'histoplasmose africaine multifocale avec une atteinte péritonéale et péricardique. Un homme de 27 ans, orpailleur, a consulté pour des lésions papuleuses et nodulaires disséminées évoluant depuis 3 mois. À l'examen, une cachexie (indice de masse corporelle 15,19kg/ $\mathrm{m}^{2}$ ), des abcès froids sur tout le tégument, des papules ombiliquées prédominant au visage et une ascite étaient notés. Les explorations paracliniques montraient une neutrophilie, une anémie, une hypoprotidémie, une ascite exsudative, une péricardite, une hypertension artérielle pulmonaire sévère, de grandes levures en forme de 8 d'Histoplasma capsulatum var. duboisii à l'examen direct du prélèvement mycologique, confirmant l'histoplasmose africaine. Litraconazole a permis d'obtenir une bonne évolution clinique. La dénutrition sévère a probablement constitué un facteur de risque de dissémination de la maladie.

Mots clés: atteinte viscérale, dénutrition, itraconazole, Burkina Faso

\section{INTRODUCTION}

Les histoplasmoses sont des mycoses dues à Histoplasma capsulatum dont il existe deux variétés: Histoplasma capsulatum var. capsulatum (Hcc), agent del'histoplasmose américaine à petites formes ou " maladie de Darling "; et l'Histoplasma capsulatum var. duboisii (Hcd), agent de l'histoplasmose dite africaine à grande forme.

L'histoplasmose africaine à Hcd se manifeste le plus souvent par une atteinte cutanée et/ou osseuse et/ ou ganglionnaire $[1,2]$. La localisation pariétale est plus rarement rapportée. Nous rapportons un cas d'histoplasmose africaine multifocale à Hcd chez un orpailleur de 27 ans caractérisé par une atteinte péritonéale et péricardique dans un contexte de dénutrition sévère diagnostiqué et pris en charge au service de dermatologie-vénérologie du CHU Yalgado Ouédraogo à Ouagadougou.

\section{OBSERVATION}

Il s'agissait d'un homme de 27 ans, orpailleur sur un site minier traditionnel, célibataire, sans antécédent pathologique connu. Il consultait pour des lésions papuleuses et nodulaires disséminées sur le tégument, évoluant depuis 3 mois sur un mode continu dans un contexte d'amaigrissement important.

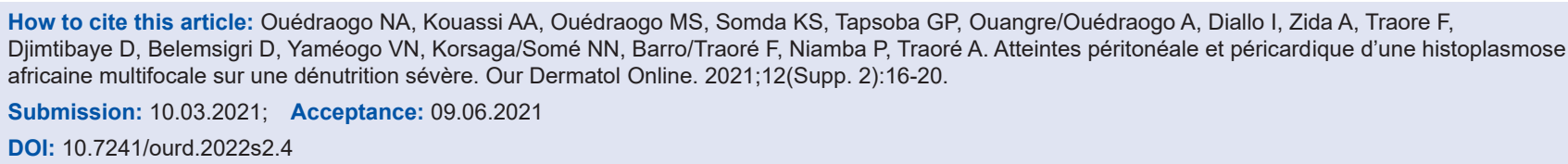


Lexamen physique, retrouvait une altération de l'état général avec une asthénie, une cachexie (indice de masse corporelle à $15,19 \mathrm{~kg} / \mathrm{m}^{2}$ ) et des odèmes des membres pelviens bilatéraux, prenant le godet et indolores.

L'examen dermatologique, relevait des abcès froids (Fig. la) sous cutanés de 2 à $4 \mathrm{~cm}$ de diamètre, de consistance ferme par endroit, mous et fluctuants en d'autres, sensibles, mobiles, disséminés sur tout le tégument et des papules hypopigmentées à centre ombiliqué, diffuses, prédominant au visage (Fig. 2a); les paumes, les plantes, les phanères et les muqueuses n'étaient pas atteintes. Le reste de l'examen notait des adénopathies cervicales, axillaires, inguinales bilatérales, mobiles, de 1 à $3 \mathrm{~cm}$ de diamètre, indolores, une ascite (Fig. 3a) avec la présence d'un signe du flot et un liquide chyleux. Il n'y avait pas de syndrome méningé, ni de signes d'atteinte pulmonaire.

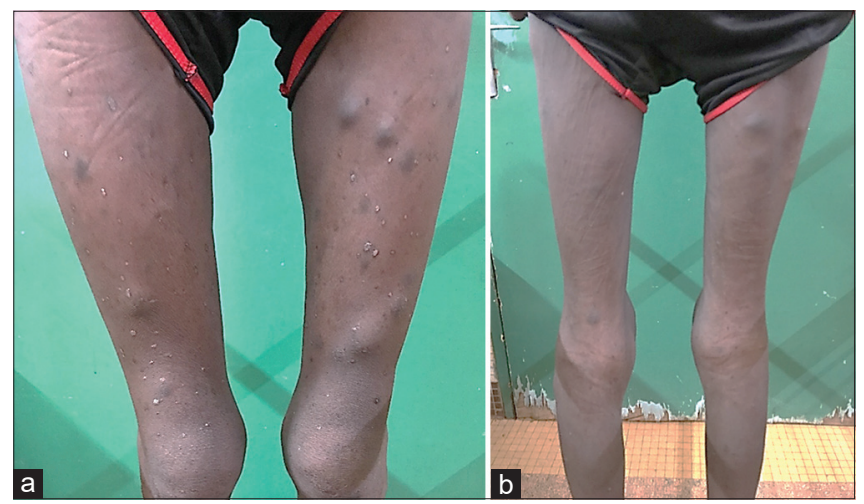

Figure 1: a: Abcès sous cutanés des cuisses [Thighs's subcutaneous abcesses]. b: Affaissement des abcès sous cutanés des cuisses [Subsidence of thighs's subcutaneous abcesses].

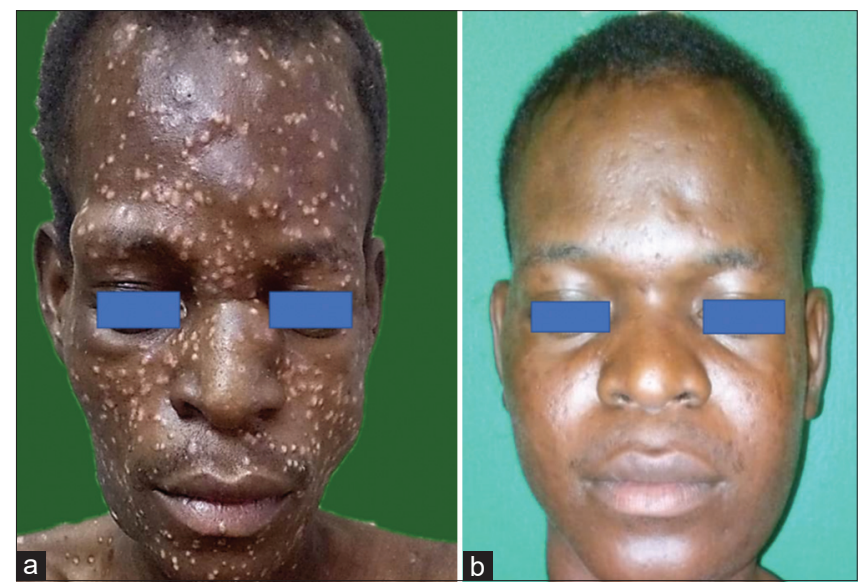

Figure 2: a: Papules ombiliquées hypopigmentéesdu visage [Ombilicated papules hypopigmented on the face]. b: Disparition des papules ombiliquées hypopigmentées du visage [Disappearance of ombilicated papules hypopigmented on the face].
Les hypothèses diagnostiques évoquées étaient une histoplasmose africaine multifocale à Hcd, une tuberculose multifocale (gommes tuberculeuses cutanées, atteinte ganglionnaire et péritonéale) et une cryptococcose multifocale.

Les explorations paracliniques retrouvaient à l'hémogramme une leucocytose à 20000 éléments $/ \mathrm{mm}^{3}$ dont 10560 neutrophiles, une anémie hypochrome microcytaire à $8,8 \mathrm{~g} / \mathrm{dl}$ d'hémoglobine et une thrombocytose à 606000 éléments/mm³. Les CD4 était à 404 cellules/ml. Les explorations biochimiques retrouvaient des troubles ioniques à type d'hyponatrémie, d'hypokaliémie, d'hypocalcémie et une hypoprotidémie sévère à $51 \mathrm{~g} / \mathrm{l}$ avec hypo albuminémie à 24,4 g/l. Les transaminases hépatiques, les bilirubines totale et conjuguée, les gamma GT, les phosphatases alcalines, la crase sanguine, la fonction rénale et la glycémie étaient normales. Les sérologies VIH 1 et 2, syphilitique, hépatiques $\mathrm{B}$ et $\mathrm{C}$ étaient négatives. Les examens cytobactériologiques et le GenXpert du pus et du liquide d'ascite à la recherche d'une tuberculose étaient négatifs.

La radiographie thoracique était normale. Léchographie abdominale objectivait une hépatomégalie et une splénomégalie homogènes et régulières ainsi qu'une ascite de grande abondance dont l'analyse cytologique révélait un exsudat.

Léchographie cardiaque révélait une péricardite avec un épanchement modéré et une hypertension artérielle pulmonaire sévère.

Lexamen mycologique direct du pus provenant des abcès froids retrouvait de grandes levures en forme de

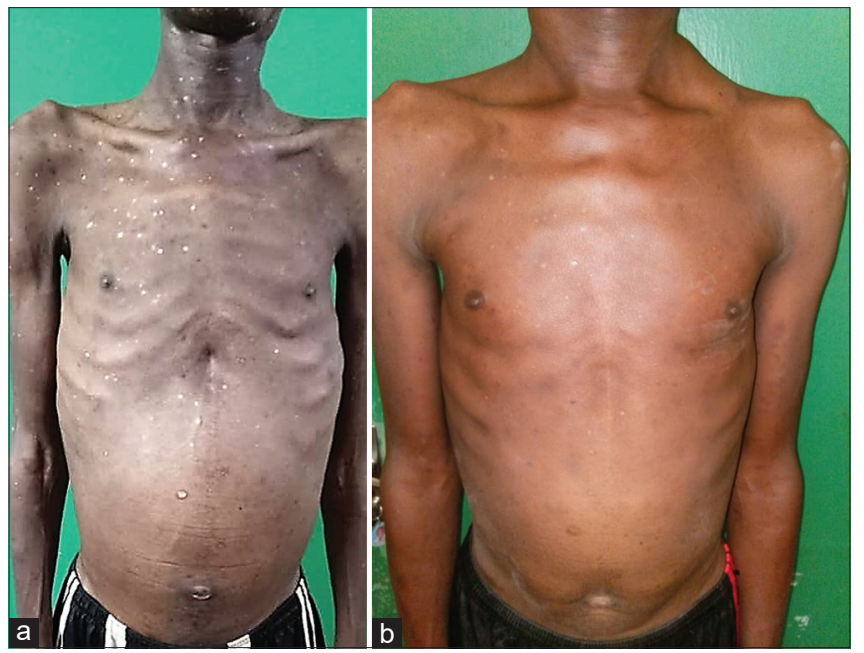

Figure 3: a: Ascite [Ascites]. b: Disparition de l'ascite après traitement [Disappearance of ascite]. 
8 d'Histoplasma capsulatum var. duboisii, confirmant l'hypothèse de l'histoplasmose africaine. Le diagnostic d'histoplasmose africaine multifocale (cutanée, ganglionnaire, viscérale, péritonéale, péricardique) sur un terrain de dénutrition sévère était retenu.

Le patient a reçu un traitement à base d'itraconazole débuté à $200 \mathrm{mg} /$ jour pendant 7 jours, puis à $400 \mathrm{mg} /$ jour en attendant d'obtenir de l'amphotéricine B, d'accessibilité difficile dans notre contexte. Il a été également mis sous spironolactone à $50 \mathrm{mg}$ /jour après deux ponctions évacuatrices de l'ascite (suivies d'une reconstitution rapide), colchicine à $\mathrm{l} \mathrm{mg} /$ jour, un régime hyperprotidique hypercalorique et des soins locaux avec un antiseptique.

Lévolution à court terme a été favorable, marquée par une disparition progressive des abcès froids (Fig. lb), un affaissement des papules (Fig. 2b), laissant des macules hypochromiques par endroits, un tarissement progressif de l'ascite (Fig. 3b) avec un périmètre abdominal passant de $80 \mathrm{~cm}$ à $72 \mathrm{~cm}$ et un gain pondéral de $3 \mathrm{~kg}$ au bout de 45 jours. Puis, l'itraconazole a été remplacé par l'amphotéricine B (obtenu gratuitement), administré par voie parentérale à dose progressive pendant 13 jours. Cependant, devant la reconstitution des abcès froids et de l'ascite, l'amphotéricine B a été arrêté et l'itraconazole réintroduit à $400 \mathrm{mg} /$ jour.

Les abcès ont régressé à nouveau avec une amélioration de l'état nutritionnel, un tarissement de l'ascite, de la péricardite et une disparition de l'hépato-splénomégalie en trois semaines. Le traitement a été poursuivi à la même dose pendant six mois et demi; durée à laquelle une disparition complète des abcès sans notion de récidive était obtenue. Le traitement a été poursuivi à demi dose soit à $200 \mathrm{mg} /$ jour pendant 18 mois.

\section{DISCUSSION}

L'histoplasmose à Hcd reste rare dans la pratique médicale quotidienne en zone subsaharienne. Des cas isolés sont le plus souvent rapportés souvent chez des patients séronégatif au VIH [1-3]. Dans la littérature, l'histoplasmose africaine semble exceptionnelle chez les patients infectés par le VIH [4].

Chez notre patient, orpailleur, la fréquentation de grottes creusées dans le sol, pouvant contenir les déjections d'oiseaux et de chauve-souris était probablement le lieu de sa contamination; bien que décrite uniquement dans la littérature pour l'Hcc.
La spécificité de notre cas repose essentiellement sur les atteintes pariétales, (péritonéale et péricardique) qui sont rarement décrites dans la littérature. En effet, des cas d'histoplasmose avec une atteinte multifocale (cutanée, ganglionnaire, hépatique, splénique et osseuse) ont été précédemment décrits à Ouagadougou $[1,5,6]$.

Bien que la précarité de vie du patient n'ai pas permis d'effectuer la recherche d'Hcd dans le liquide d'ascite et de péricardite, la bonne évolution clinique sous traitement plaide en faveur de cette hypothèse.

Devant cette ascite exsudative de grande abondance, associée à des lésions cutanées à type d'abcès froids évoquant des gommes et des adénopathies multifocales non inflammatoires, nous avons discuté une tuberculose, du fait de la présence de cette dernière en mode endémique dans le pays. Cependant, la négativité de la recherche du gène du bacille de Kock, du GenXpert nous ont permis d'écarter cette hypothèse.

Devant les papules ombiliquées, la cryptococcose disséminée pouvait être évoquée [7], mais l'absence d'une atteinte pulmonaire, l'absence de foyer alvéolaire et interstitiel, ainsi que de cryptococcomes à la radiographie pulmonaire, l'absence d'une atteinte neuro-méningée ont permis d'éliminer cette hypothèse.

Létat de dénutrition sévère avec l'hypoalbuminémie à 24,4 g/l, considérée comme un facteur de mauvais pronostic, a probablement favorisé la dissémination de la maladie chez notre patient.

Pour traiter l'histoplasmose disséminée, l'amphotéricine $\mathrm{B}$ et l'itraconazole sont préconisés. Le fluconazole à fortes doses peut également être utilisé [8]. Du fait de la disponibilité irrégulière de l'amphotéricine $\mathrm{B}$ dans notre contexte, et de la reprise des lésions sous cette molécule, nous avons finalement opté pour l'itraconazole. Lamélioration du tableau clinique sous itraconazole nous permet d'affirmer que cette molécule est une alternative intéressante dans notre contexte de travail.

\section{CONCLUSION}

Les atteintes viscérale et pariétale de l'histoplasmose africaine existent, simulant entre autres une tuberculose multifocale. La dénutrition sévère peut constituer un facteur de risque de dissémination de 
la maladie. Litraconazole est efficace, de maniement et d'accessibilité plus aisés dans notre contexte que l'amphotéricine B.

\section{RÉFÉRENCES}

1. Barro/Traoré F, Sanwidi M, Dao F, Korsaga/Somé N, Niamba P, Traoré A, et al. Histoplasmose africaine disséminée chez un enfant immunocompétent au Burkina Faso: un cas. Our Dermatol Online. 2013;4:361-8.

2. Savi De Tove YS, Ogouyemi-Hounto A, Azon-Kouanou A, Savi de Tove K-M, Atadokpèdé F, Kouessi Agbodandé A, et al. Histoplasmose africaine: difficultés de prise en charge: à propos de 2 cas diagnostiques au Bénin. J Mycol Med. 2017;27:e34.

3. Diadié S, Ahy Diatta B, Ndiaye M, Gaye M, Sow D, Ndiaye NT, et al. Histoplasmose multifocale à Histoplasma capsulatum Var. duboisii chez un Sénégalais de 22 ans sans immunodépression prouvée. J Mycol Med. 2016;26:265-70.

4. Fernandez C, Gazaille V, Werbrouck-Chiraux A, Belmonte O, Sultan-Bichat N, Agape P, et al. [American histoplasmosis: clinicopathological features in immunocompetent and immunocompromised patients. A report of two cases of infection by Histoplasma capsulatum on Reunion Island]. Rev Mal Respir. 2014;31:447-53.

5. Ouedraogo NA, Nde/Ouedraogo NA, Tiemtore-Kambou BMA, Traore F, Ouedraogo Ms, Tapsoba GP, et al. Histoplasmose africaine à localisation multifocale sur terrain immunocompétent au VIH. Ann Univer Ouaga I Pr Joseph Ki_Zerbo. 2016;Serie D;17:229-38.

6. Zida A, Niamba P, Barro-Traoré F, Korsaga-Somé N, Tapsoba F, Briegel J, et al. Disseminated Histoplasmosis caused by Histoplasma capsulatum var. duboisii in a non-HIV patient in Burkina Faso: Case Report. J Med Mycol. 2015;25:159-62.

7. Kumar Yadalla HK, Rama Rao GR. Cutaneous Cryptococcosis: a marker of life threatening disseminated cryptococcosis in HIV AIDS. Our Dermatol Online. 2011;2:204-7.

8. Mandengue Ebenye C, Takuefou Mfangan B, Nouédoui C, Atangana PJA. Histoplasmose disséminée traitée par bolus de fluconazole. Med Santé Trop. 2015;25:110-1.

Copyright by Nomtondo Amina Ouédraogo, et al. This is an open-access article distributed under the terms of the Creative Commons Attribution License, which permits unrestricted use, distribution, and reproduction in any medium, provided the original author and source are credited.

Source of Support: Nil, Conflict of Interest: None declared. 